\title{
Characterization of Gelatin-Carboxymethylcellulose Scaffolds
}

\author{
Fasai Wiwatwongwana and Nattawit Promma
}

\begin{abstract}
Scaffold is a biocompatible material that helps relieve patients with skin loss symptoms caused by, for example, burns and ulcer. The scaffold also provides suitable condition at the wounded site and promotes faster healing process. In this research, gelatin was selected for scaffold fabrication with additional Carboxymethylcellulose (CMC) for the structural strengthening where freeze drying method was used to form the porous structure. The scaffold was fabricated in various gelatin-CMC ratio for the investigation which was 100:0, 90:10, $80: 20,70: 30$ and 60:40. The material behavior of this scaffold is likely to be a foam-like hyperelastic material. Therefore, large deformation theory was applied to achieve the engineering stress constitutive equation in forms of Blatz-Ko model. The large deformation theory has been used to derive the constitutive equation to obtain the engineering stress equation in the form of Blatz-Ko hyperelastic model. The mechanical characterization of the scaffold was done by performing compressive test using universal testing machine (UTM). The data obtained from the UTM were used to plot the stress-strain relation. The identification of shear modulus of the scaffold was done using curve fitting method where it is approximately $\mathbf{7 \%}$ according to the Blatz-Ko model description that it is suitable for infinitesimal strain theory. The physical characterization was done by using scanning electron microscopy (SEM) to investigate pore size of scaffolds. The results obtained showed the appropriate pore size of the scaffold with average pore size of $130 \mu \mathrm{m}$ to $180 \mu \mathrm{m}$.
\end{abstract}

Index Terms-Hyperelastic material, Blatz-Ko, shear modulus, scaffold, gelatin, carboxymethylcellouse.

\section{INTRODUCTION}

The aim of tissue engineering is a science of constructing biological structure to support their task. One of the most common injuries caused by diseases and accidents is skin loss. Skin loss symptoms can be categorized in many levels and different causes such as burns, ulcers and diabetics. The treatment using skin replacement material is also widely available. The purpose of scaffold is the wound enclosure to prevent infection and accelerate the wound healing. The skin replacement material is a biomaterial and biocompatible material as the real skin, called a "scaffold" [1], [2], which

Manuscript received January 30, 2018; revised January 23, 2019. This work was supported in part by National Science and Technology Development Agency, Ministry of Science and Technology, Thailand; Department of Mechanical Engineering, Faculty of Engineering; Dental Material Laboratory, Faculty of Dentistry; Faculty of Associated Medical Science, Chiang Mai University.

Fasai Wiwatwongwana is with Department of Advanced Manufacturing Technology, Faculty of Engineering, Pathumwan Institute of Technology, 833 Rama 1 Road, Wangmai, Pathumwan, Bangkok 10330, Thailand (e-mail fasaiw227@gmail.com).

Nattawit Promma is with Department of Mechanical Engineering, Faculty of Engineering, Chiang Mai University, 239 Huay Kaew Road, Muang District, Chiang Mai 50200, Thailand (e-mail: nano_504@hotmail.com). allows the skin to reproduce in a suitable condition and heal completely. However, the effective tissue engineering and scaffold technology are expensive. Therefore, the affordable scaffold is also one of the purposes of this research.

The development of the skin replacement material that supports the recovery mechanism is one of the challenges in scaffold fabrication. The design of the scaffold can be varied depending on the application of usage and it could be either derived naturally or synthetically [3], [4]. The main characteristic that a scaffold must have is suitable strength (mechanical characteristic) and pore size (physical characteristic) where both are conducted in this research. In the previous research, the biocompatibility has been confirmed. In terms of mechanical characteristic, the material behavior of the scaffold has to be examined to verify its behavior. Hyperelastic material is a material subjected to large deformation and it could act as a rubber-like or foam-like material. In this research, Blatz-Ko model is used because it is suitable for foam-like material and because skin can subject to both axial and shear force, the identified parameters are young's modulus and shear modulus. The scaffold requires stability and relatively affordable.

Scaffold made from gelatin has shown to be positively interacted with cells which have a research approval of in vitro biocompatibility test of gelatin with fibroblast cells [5], [6]. The cells showed a good affinity and proliferation on the gelatin scaffolds after 14 days of culturing without any signs of biodegradation [7]. The second biological material used to blend with gelatin scaffold which can improve strength of scaffold structure is carboxymethylcellulose (CMC). CMC is a derivative of cellulose by reacted with sodium hydroxide and chloroacetic acid. The properties of CMC are good in viscosity building, flocculation and high shear stability. CMC is available and easily purchased. It is very cheap compared to other polysaccharides [8], [9]. Therefore, gelatin and Carboxymethylcelluose (CMC) are selected to fabricate a porous tissue engineering scaffold. Furthermore, the scaffolds are treated with various treatment methods for material strengthening which are dehydrothermal treatment and chemical treatment [10].

\section{MATERIAL FABRICATION}

From the previous research on the dehydrothermal scaffolding, the scaffold fabrication procedures used are the same [11]. The scaffold is made of gelatin where CMC is added for strength improvement. Type A gelatin was purchased from BIO BASIC INC, Canada. It is a reagent grade and derived from pork skin with bloom number of 240-270 and $\mathrm{pH} 4.5-5.5$ at $25^{\circ} \mathrm{C}$. Its viscosity was 3.5-4.5 cps 
and moisture less than $12.0 \%$ and carboxymethylcellulose sodium salt (CMC) was purchased from Sigma-Aldrich, St. Louis, MO, USA. It has medium viscosity of 400-800 cps in a $2 \%$ aqueous solution at $25{ }^{\circ} \mathrm{C}$. The gelatin-CMC solution is made using deionized water. The scaffold are made in five different gelatin-CMC ratios which are 100:0, 90:10, 80:20, 70:30 and 60:40. The gelatin-CMC scaffold fabrication is done by preparing gelatin solution by mixing gelatin powder to deionized water (DI water, $0.8 \mathrm{wt}$. \%) then leave it at room temperature for 1 hour before stir it at $50^{\circ} \mathrm{C}$ for another 1 hour while CMC solution is done by mixing CMC and DI water together and stirring at $70{ }^{\circ} \mathrm{C}$ for 30 minutes. Then blending gelatin and CMC solution at gelatin-CMC ratio of 100:0, 90:10, 80:20, 70:30 and 60:40 then stir it at $50^{\circ} \mathrm{C}$ for 15 minutes each. We pipette the solution into 24-well culture plate with volume $1 \mathrm{ml}$ per well and freeze them for more than 12 hours in a $-20^{\circ} \mathrm{C}$ condition. The scaffold is placed into a Lyophilizer (Freeze-Dry Machine) at $-50{ }^{\circ} \mathrm{C}$ for 24 hours before label them and store all scaffolds in a humid controlled container.

\section{MAterial PARAMETER IDENTIFICATION}

\section{A. Physical Property Identification}

The fabrication method of this scaffold is mainly using freeze-drying technique where porous structure is formed. Scanning Electron Microscopy (SEM, JEOL: JSM-5910 LV SEM) was used to determine the pore size of scaffold. All scaffolds were investigated using 60x magnifications and $15 \mathrm{kV}$ voltage acceleration.

\section{B. Mechanical Properties Identification}

The compressive testing was performed by using universal testing machine (UTM) with compression rate of 0.1 $\mathrm{mm} /$ minute in dry condition at $25{ }^{\circ} \mathrm{C}$ to collect load-deformation data from the experiment to obtain stress-strain information. The compression rate at 0.1 $\mathrm{mm} /$ minute referenced from [12] and used to prove that at the same testing condition the Blatz-Ko model can fit better that the Neo-Hokean model. The sample used for each ratio of scaffold was 5 samples $(n=5)$. The shear modulus identification of the material is identified by Blatz-Ko hyperelastic using curve fitting method. The tested scaffolds were divided into 5 mixtures which are pure gelatin, 90:10, 80:20, 70:30 and 60:40 gelatin-CMC.

The finite-strain theory or large deformation theory deals with arbitrarily large rotations and strain of deformed material. It is applicable for the hypereleastic material behavior description and commonly suitable for soft tissue description [13]. Strain energy potential function is a scalar function of deformation tensor and derived using strain to determine stress, for Eulerian configuration (Deformed configuration). The Piola-Kirchhoff stress tensor, in hyperelastic, can be expressed to determine components of the Cauchy stress tensor in terms of the principle invariants of the left Cauchy-Green deformation tensor, the Cauchy stress components, showed in (1) [14]. Generally, the Cauchy stress is expressed in both $\mathrm{i}$ and $\mathrm{j}$ direction. In order to avoid the pressure term, the difference between $\sigma_{\mathrm{i}}-\sigma_{\mathrm{j}}$ and given $\sigma_{\mathrm{j}}$ are zero.

$$
\sigma=-p I+2 \frac{\partial W}{\partial r_{1}} B-2 \frac{\partial W}{\partial r_{2}} B^{-1}
$$

where $w$ represents the strain energy function per unit initial volume, $\sigma$ represents the Cauchy stress tensor, $B$ represents the left Cauchy-Green deformation tensor, $I$ represents the principle invariant and p represents pressure value.

In the uniaxial extension case, the extension occurs along the lateral, $i$, direction and the gradient deformation can be written in form of principle stretch $(\lambda)$. From (1), writing Cauchy stress equation in two directions $\left(i_{s} j\right)$ and subtract each other in order to obtain the Cauchy stress equation in uniaxial extension (2). An engineering stress $(T)$ can be obtained by dividing (2) by $\lambda$ as shown in (3).

$$
\begin{aligned}
& \sigma_{11}=2\left(\lambda^{2}-\frac{1}{\lambda}\right)\left(\frac{\partial W}{\partial r_{1}}+\frac{1}{\lambda} \frac{\partial W}{\partial r_{2}}\right) \\
& T_{11}=2\left(\lambda-\frac{1}{\lambda^{2}}\right)\left(\frac{\partial W}{\partial r_{1}}+\frac{1}{\lambda} \frac{\partial W}{\partial r_{2}}\right)
\end{aligned}
$$

When the principle stretches $(\lambda)$ have the value of $\varepsilon+1$, the term $I_{i}$ is the invariant that can be calculated and written as shown in (4).

$$
I_{1}=\lambda^{2}+\frac{2}{\lambda}, I_{2}=\frac{1}{\lambda^{2}}+2 \lambda, I_{a}=1
$$

Blatz-Ko model describes the material behavior that acts as foam rubber and applicable to both cases of compressible and incompressible material [15]. Using Blatz-Ko model as incompressible material, after the derivation, it is apparently shows the equation of Neo-Hookean model. However, in compressible case, the strain energy equation where Poisson ratio is $0.25(v=0.25)$ can be expressed as shown in (5).

$$
W\left(I_{1}, I_{2}, I_{a}\right)=\frac{\mu}{2}\left(\frac{I_{2}}{I_{3}}+2 I_{a}^{\frac{1}{2}}-5\right)
$$

Then the engineering stress equation in term of Blatz-Ko compressible case can be obtained as shown in (6).

$$
T_{11}=\mu\left(1-\frac{1}{\lambda^{3}}\right)
$$

From the data of stress-strain curve obtained from an experiment, the curve fitting using the function derived from Blatz-Ko hyperelastic model. The calculation method, in this research, is nonlinear least-squared. The shear modulus is obtained earlier from curve fitting method; therefore, the young's modulus (E) can be also obtained from (7) [16].

$$
\mathrm{G}=\frac{\mathrm{B}}{2(1+\mathrm{w})}
$$

\section{RESULT}

\section{A. Mechanical Characterization Experimental Result}

For each type of scaffolds, there are 5 samples to be tested. 
The Curve fitting method was being used to determine the parameter of the material using Blatz-Ko model. Fig. 1 is the examples showing the stress-strain curve and curve fitting of gelatin-CMC scaffold at ratios 80:20.

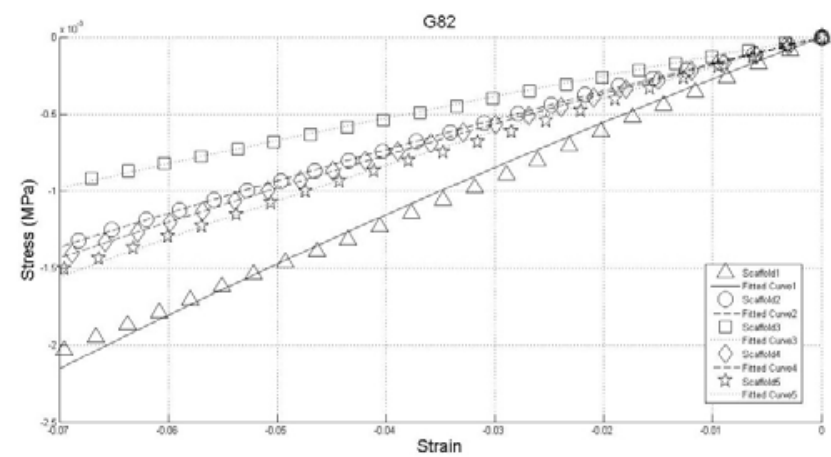

Fig. 1. Curve fitting of scaffolds.

From the results, Blatz-Ko model can fit the curve at $7 \%$ strain. Each type of scaffolds was plotted containing 5 samples. Each sample was represented by different kinds of marker. The curve was fitted and shown as a line. The value shown in Table I is the average value of each scaffold where the average $r$ square of each case is more than 0.99 .

TABLE I: CURVE FITTING RESULTS.

\begin{tabular}{|c|c|c|c|}
\hline Scaffolds & $\begin{array}{l}\text { Average Shear } \\
\text { Modulus (kPa) }\end{array}$ & SD & $\begin{array}{l}\text { Average } \\
\text { Young's } \\
\text { Modulus (kPa) }\end{array}$ \\
\hline G100 & 7.73 & 1.73 & 19.32 \\
\hline G91 & 9.21 & 1.91 & 23.04 \\
\hline G82 & 6.15 & 1.56 & 15.39 \\
\hline G73 & 4.81 & 1.04 & 12.03 \\
\hline G64 & 4.91 & 0.36 & 12.27 \\
\hline
\end{tabular}

The summary of the scaffolds' shear modulus is shown in Fig. 2 where the gelatin-CMC at ratio of 100:0, 90:10, 70:30, 80:20 and 60:40 represented by the labels G100, G91, G82, G73 and G64, respectively. The scaffolds are represented by circle marker. From this figure, it shows that the G91 scaffold gives higher value of shear modulus.

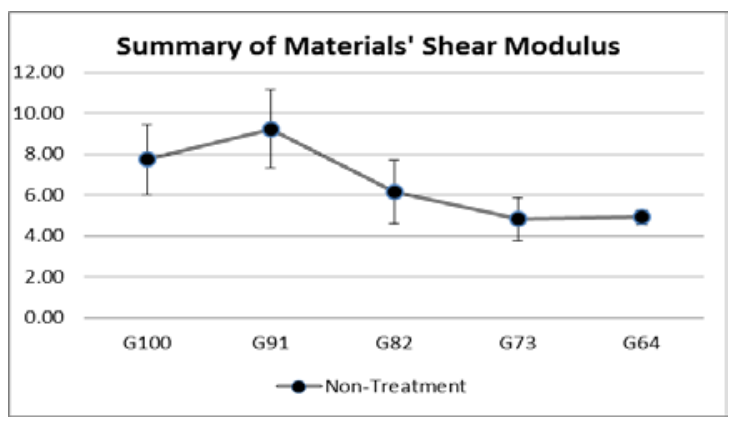

Fig. 2. Summary of shear modulus of scaffolds.

\section{B. Physical Characterization Experimental Result Non-Treatment Scaffolds}

The cross-sectional structures of scaffolds are taken and shown in Fig. 3. The scaffolds are considered as a homogeneous porous structure, except G100 (Fig. 3(a)) where it can be considered as non-homogeneous porous structure.

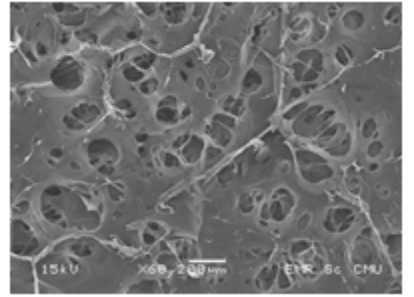

(a)

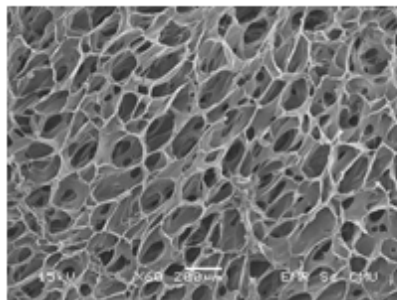

(c)

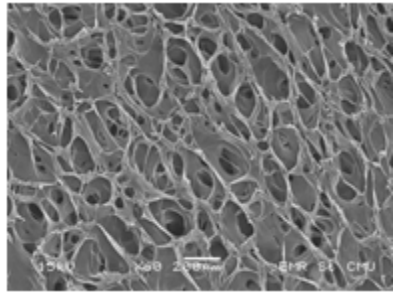

(b)

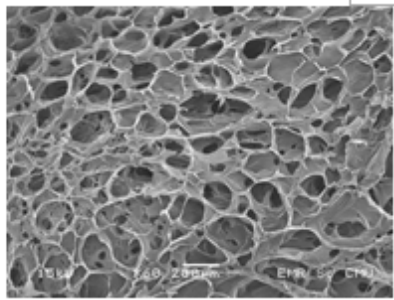

(d)

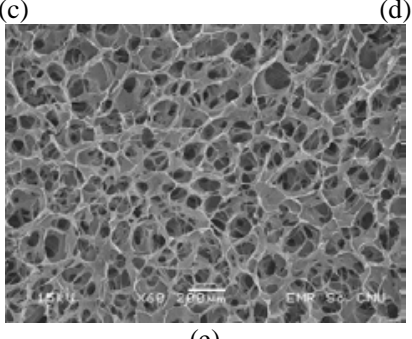

(e)

Fig. 3. SEM of non-treatment scaffolds (a) G100 (b) G91 (c) G820020(d) G73 and (e) G64.

The average pore size of the scaffolds is shown in Table II. The gelatin-CMC at 100:0, 90:10, 70:30, 80:20 and 60:40 ratio represented by the labels G100, G91, G82, G73 and G64, respectively. The pore size ranges approximately between $130 \mu \mathrm{m}$ to $180 \mu \mathrm{m}$ which is in the appropriate range of a skin tissue engineering scaffold.

\begin{tabular}{ccc}
\multicolumn{3}{c}{ TABLE II: AverAGE PORE SizE OF SCAFFOLDS } \\
\hline \hline Scaffolds & Average Pore Size $(\mu \mathrm{m})$ & SD \\
\hline G100 & 130.21 & 36.17 \\
G91 & 162.55 & 51.49 \\
G82 & 180.00 & 35.74 \\
G73 & 156.60 & 36.60 \\
G64 & 165.11 & 42.55 \\
\hline \hline
\end{tabular}

\section{DISCUSSION}

This research suggests that CMC may only be useful as a strengthening agent for gelatin scaffolds at a narrow concentration range. Further research should be focused on the various treatment methods for scaffold strengthening which are dehydrothermal treatment and chemical treatment. The appropriate freeze-drying time for each scaffold could be identical. Therefore, the specific suitable freeze-drying time for each type of scaffold should be determined. This may allow each type of scaffold to obtain maximum strength of material. The Blatez-Ko model used in this research can fit the curve at $7 \%$ according to the description of this model where it is suitable in infinitesimal strain situation. For further investigation, more complex model, such as Ogden can be used in case of foam-like material. Moreover, a higher number of samples and repetition are encouraged in order to improve experimental accuracy. The stress-strain curve of the scaffolds are scattered, so more samples may be resulted 
in more accurate outcome. The porosity and biodegradability experiment should be performed. Moreover, since the scaffold will be used in wet condition, its mechanical properties in wet condition should also be identified.

\section{CONCLUSION}

A scaffold for skin is used to treat patients with skin loss symptoms. However, the price of commercially products remains expensive. This research aims to find new inexpensive material as an alternative. The scaffold is made of gelatin where CMC is selected as the scaffold strengthener. CMC is solved into gelatin solution forms the scaffold at various gelatin-CMC ratio as following; 100:0, 90:10, 80:20, $70: 30$ and 60:40. The physical characterization, in this research, is the investigation of pore size by using scanning electron microscopy (SEM). The scaffolds are randomly selected from each type and ratio to be investigated. The scaffolds have homogeneous structure except G100 which can be considered as non-homogeneous porous structure. The average pore sizes of all scaffolds range from $130 \mu \mathrm{m}$ to $180 \mu \mathrm{m}$ which are considered as appropriate range of pore size. For the mechanical characterization, the scaffolds are compressed to $70 \%$ deformation. The Blatz-Ko model is used to identify the shear modulus by using curve fitting method. The result shows the Blatz-Ko model can fit the curve at $7 \%$ strain which is according to the characteristic of this model (infinitesimal strain). The maximum shear modulus of scaffold is from gelatin-CMC ratio at 90:10 which is $9.21 \pm 1.91 \mathrm{kPa}$. The maximum shear modulus obtained from gelatin-CMC ratio at $80: 20$ is $28.12 \pm 8.43 \mathrm{kPa}$ and the maximum young's modulus also obtained is approximately $23.04 \mathrm{kPa}$. Accordingly, CMC is the strengthening compound and resulted in increasing the strength of the gelatin scaffold. However, the higher CMC content is not significantly effect on material strengthening. This can be implied that at only a certain amount of CMC can increase the strength of the scaffold and improper portion of CMC and treatment may not help the strength improvement.

\section{ACKNOWLEDGMENT}

This research conducted under the support of National Science and Technology Development Agency, Ministry of Science and Technology, Thailand; Department of Mechanical Engineering, Faculty of Engineering; Dental Material Laboratory, Faculty of Dentistry; Faculty of Associated Medical Science, Chiang Mai University. It was gratefully acknowledged for providing facilities and experiment being required for this research.

\section{REFERENCES}

[1] P. X. Ma and J. Elisseeff, Scaffolding in Tissue Engineering, Florida, U.S.: CRC Press, 2006, ch. 1, pp. 3-10.

[2] P. X. Ma, “Scaffolds for tissue fabrication,” Materials Today, pp. 30-40, 2004.

[3] J. O. Hollinger, An Introduction to Biomaterials, 2nd ed., U.S.: CRC Press, 2012, ch.2, pp. 7-14.

[4] J. B. Park and J. D. Bronzino, Biomaterials: Principles and Applications, U.S.: CRC Press, 2002, ch.7, pp. 141-172.

[5] Y. Tabata, A. Nagano, M. Muniruzzaman, and Y. Ikada, "In vitro sorption and desorption of basic fibroblast growth factor from biodegradable hydrogels,” Biomaterials, vol. 19, pp. 1781-1789, 1998.
[6] S. B. Lee, H. W. Jeon, Y. W. Lee, Y. M. Lee, K. W. Song, M. H. Park, Y. S. Nam, and H. C. Ahn, "Bio- artificial skin composed of gelatin and (1-3), (1-6) - $\beta$-glucan,” Biomaterials, vol. 24, pp. 2503-2511, 2003.

[7] S. B. Lee, Y. H. Kim, M. S. Chong, S. H. Hong, and Y. M. Lee, “Study of gelatin-containing artificial skin V: Fabrication of gelatin scaffolds using a salt-leaching method,” Biomaterials, vol. 26, pp. 1961-1968, 2005.

[8] D. Capitani, F. Porro, and A. L. Segre, "High field NMR analysis of the degree of substitution in carboxymethyl cellulose sodium salt," Carbohydrate Polymers, vol. 42, pp. 283-286, 2000.

[9] D. R. Biswal, and R. P. Singh, "Characterisation of carboxymethyl cellulose and polyacrylamide graft copolymer," Carbohydrate Polymers, vol. 57, pp. 379-387, 2004.

[10] L. H. H. Olde Damink, P. J. Dijkstra, M. J. A. van Luyn, P. B. van Wachem, P. Nieuwenhuis, and J. Feijen, "Cross-linking of dermal sheep collagen using a water-soluble carbodiimide,” Biomaterials, vol. 17, pp. 765-773, 1996.

[11] N. Siroros and N. Promma, "Determination of material parameter of gelatin-carboxymethylcellouse scaffold with dehydrothermal crosslinking technique using curve fitting method," Advanced Materials Research, vol. 931-932, pp. 375-380, 2014.

[12] F. Wiwatwongwana, Y. Khunathon, W. Rangsri, N. Promma, and S. Pattana, "Identification of shear modulus of gelatin blended with carboxymethylcellulose scaffolds using curve fitting method from compressive test,” Journal of Materials Science Research, vol. 1, no. 4, pp. 106-113, 2012.

[13] G. A. Holzapfel, Nonlinear Solid Mechanics: A Continuum Approach for Engineering, West Sussex, England: John Wiley \& Sons, Ltd., 2000, ch. 6, pp. 205-304.

[14] N. Promma, B. Raka, M. Grediac, E. Toussaint, J.-B. Le Cam, X. Balandraud, and F. Hild, "Application of the virtual fields method to mechanical characterization of elastomeric materials,” International Journal of Solids and Structures, vol. 46, pp. 698-715, 2009.

[15] P. J. Blatz, and W. L. Ko, "Application of finite elastic theory to the deformation of rubbery materials," Transactions of the Society of Rheology, vol. 6, pp. 223-251, 1962.

[16] F. P. Beer, E. R. Johnston, JR. and J. T. Dewolf, Mechanics of Materials, 4th ed., New York, USA: McGraw-Hill, 2006, ch. 6, pp. 372-413.

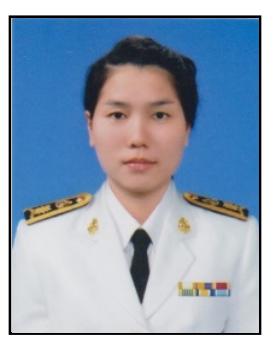

Fasai Wiwatwongwana was born on November 5, 1982 in Chiang Mai Province, Thailand. About her education, she graduated Ph.D. of Mechanical Engineering in December 2012 from Chiang Mai University, Chiang Mai, Thailand, Master of Chemical Engineering in April 2007 from Chulalongkorn University, Bangkok, Thailand and Bachelor of Engineering, Chemical Engineering in April 2004 from Thammasat University, Bangkok, Thailand. Her phone number is +66-2104-9099 ext. 3080, 3081 and mobile number is +66-86-830-3949. She has teaching experiences as a lecturer at Department of Manufacturing Engineering, Faculty of Engineering, Pathumwan Institute of Technology from June 2014 to present, Department of Mechanical Engineering, Faculty of Engineering, Rajamangala University of Technology Lanna Chiang Mai from June 2013 to May 2014 and Biomedical Engineering Program (International Program), Graduate School, Chiang Mai University from June 2009 to May 2013. She also has experiences as a researcher at Biomedical Engineering Center, Chiang Mai University from March 2008 to May 2013 and Center for Agricultural Biotechnology, Faculty of Agriculture, Chiang Mai University from July 2007 to February 2008.

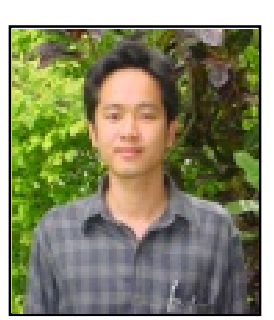

Nattawit Promma was born on July 15, 1977 in Chiang Mai Province, Thailand. About his education, he received his Bachelor degree of Mechanical Engineering in May 1999 and Master of Energy Engineering in October 2001 from Chiang Mai University, Chiang Mai, Thailand. After graduation, he was employed under the position of engineer by Fujikura Thailand Co., Ltd. for 4 months. He served in position of lecturer at Department of Mechanical Engineering, Faculty of Engineering, Chiang Mai University in 2002. His phone number is +66-5394-4145 ext. 936 and mobile number is +66-81-536-4244. In 2009, he graduated Ph.D. of Mechanical Engineering in April 2009 from Blaise Pascal University - Clermont Ferrand II, France. He is an assistant professor in Mechanical Engineering at Department of Mechanical Engineering, Faculty of Engineering, Chiang Mai University at present. 